\title{
William H. Davenport, Santa Cruz Island, Figure Sculpture and Its Social and Rituel Contexts
}

University of Pennsylvania, Museum of Archaeology and Anthropology, Philadelphia, 2005

\section{Gilles Bounoure}

\section{(2) OpenEdition}

Journals

Édition électronique

URL : http://journals.openedition.org/jso/661

DOI : $10.4000 /$ jso.661

ISSN : $1760-7256$

Éditeur

Société des océanistes

Édition imprimée

Date de publication : 1 décembre 2006

Pagination : 211-213

ISSN : 0300-953x

Référence électronique

Gilles Bounoure, «William H. Davenport, Santa Cruz Island, Figure Sculpture and Its Social and Rituel Contexts », Journal de la Société des Océanistes [En ligne], 122-123 | Année 2006, mis en ligne le 23 avril 2008, consulté le 21 septembre 2020. URL : http://journals.openedition.org/jso/661 ; DOI : https:// doi.org/10.4000/jso.661 
Stéfani. L'ouvrage est divisé en trois grandes parties, comme l'était l'exposition de Chartres. Les promoteurs de cette exposition ont fait ce choix pour évoquer la situation de l'île de 'Uvea dans son contexte régional, la préhistoire et l'histoire coloniale de cette île, la société et la culture actuelle, telle qu'elle se présente à la fin $\mathrm{du} \mathrm{Xx}^{\mathrm{e}}$ siècle. Chacune de ces parties est constituée de textes réalisés par des universitaires archéologue, anthropologue, historien, linguiste, muséologue..., suivies par un volet catalogue des œuvres présentées dans l'exposition (pp. 25-36, pp. 57-66 et pp. 121-154). Si la majorité des photos qui illustrent ces objets sont en noir et blanc, il est heureux que les auteurs aient choisi la couleur pour présenter les plus beaux tapa (pp. 24, 26, 27, 87, 89, 93).

Dans son introduction, Claude Stéfani note que le recollement des collections uvéennes dans les musées français est loin d'être terminé, vu la difficulté à identifier les objets provenant précisément de 'Uvea. Outre les deux fonds qui ont servi de base à l'exposition, des collections substantielles existent également dans les musées d'Angoulème, Dunkerque, Grenoble, La Neylière, Lille, Lyon et La Rochelle. Le fonds du gouverneur Louis-Joseph Bouge de Chartres est l'un des plus structurés et des plus documentés. Claude Stéfani fait très justement remarquer qu'il est difficile de définir la provenance exacte des pièces de Polynésie occidentale. En effet, depuis des siècles, les peuples des îles de Fidji, Futuna, Nuie, Rotuma, Samoa, Tonga, Tokelau, Tuvalu et 'Uvea-Wallis n'ont cessé de s'échanger des objets d'usage quotidien. C'est la raison pour laquelle des pièces originaires de ces diverses îles ont été également présentées dans l'exposition (pp. 26-33). On notera cependant l'absence dans les collections uvéennes de sculptures anthropomorphes en bois ou en ivoire sculptés ${ }^{8}$ comme il en existe dans les collections tongiennes ou fidjiennes. Dans cette région du Pacifique, les cultures des divers archipels se sont influencées les unes les autres et constituent « un parfait exemple de zone d'échanges culturels intenses » (p. 10).

La réussite de l'exposition du musée des BeauxArts de Chartres, 'Uvea-Wallis, une île pêchée par les dieux, comme de son catalogue, doit beaucoup au talent et à la personnalité de Claude Stéfani (muséologue) et d'Hélène Guiot (ethno-archéologue). Sa connaissance du terrain, où elle a passé plusieurs années, lui a permis de choisir des auteurs et des textes qui dans leur grande majorité accompagnent très bien les objets présentés pour faire découvrir aux lecteurs la culture de 'Uvea-Wallis. Les documents historiques reproduits viennent compléter la description $\mathrm{du}$ contexte dans lequel les objets ont été collectés sur le terrain. C'est le cas des extraits de récits des découvreurs: Robertson sur l'expédition du Dolphin en 1767, Maurelle sur le voyage de la Princesse en 1781 et Edwards sur celui de la Pandora en 1791 (pp. 46-47). Ces textes relatent les premiers contacts entre les Européens et les peuples de 'Uvea-Wallis. La liste d'inventaire d'une douzaine d'objets provenant de l'institution missionnaire de l'Guvre de la Propagation de la
Foi à Lyon (p. 13) apporte quelques informations supplémentaires. On aurait cependant aimé en savoir plus sur les objets uvéens se trouvant dans des musées autres que français, Claude Stéfani évoquant seulement les objets uvéens du Bishop Museum d'Honolulu collectés par l'ethnologue américain Edwards Burrows (p. 13).

Les photos de terrain en couleur viennent compléter agréablement les textes et accompagner les photos d'objets du catalogue. Il aurait été judicieux de préciser un peu plus les identifications des matériaux constituant les pièces présentées et d'en décrire brièvement les techniques de mise en œuvre. Un lexique uvéen et une bibliographie sommaire concluent cet ouvrage de référence sur l'île de 'Uvea-Wallis, particulièrement pour le fonds uvéen du musée de l'Homme et celui du musée national des Arts africains et océaniens qui se trouvent actuellement réunis dans le nouveau musée du quai Branly qui a ouvert ses portes fin juin 2006. L'ensemble de la collection d'objets uvéens y sera donc prochainement accessible à tous et visible virtuellement sur la banque de données du musée.

Christian COIFFIER

département Océanie, musée de l'Homme (MNHN)

\section{William H. Davenport, 2005. Santa Cruz Island, Figure Sculpture and Its Social and Rituel Contexts, Philadelphia, University of Pennsylvania Museum of Archaeology and Anthropology, ouvrage relié sous jaquette avec photos couleurs, bibliographie, index, illustrations en noir et blanc (22 illustrations dans le texte et 55 planches) et CD reproduisant 44 planches couleur, cartes, XIV et 234 p.}

Deux chercheurs auront plus que d'autres contribué, dans la seconde moitié du $\mathrm{Xx}^{\mathrm{e}}$ siècle, à éclairer les traditions culturelles de l'archipel de Santa Cruz (aujourd'hui Temotu, la province la plus à l'est de l'État des Salomon indépendantes) et spécialement de son île principale, Ndende, Gerd Koch (1922-2005) et William Davenport (1922-2004), l'auteur de cet ouvrage posthume. Exactement contemporains, ayant eu l'un comme l'autre à affronter les difficultés de la Grande Crise puis les périls de la Deuxième Guerre mondiale, l'Allemand et l'Américain avaient beaucoup de traits en commun, et notamment une même passion de la mer et de la navigation à l'ancienne, qui a certainement compté dans leur vocation d'océanistes. Gerd Koch, dont j'ai évoqué récemment les travaux et la personnalité hors du commun (JSO 120-121), n'a effectué dans cet archipel qu'une seule mission (novembre 1966 - février 1967) aux résultats abondamment publiés, tandis que William Davenport y a multiplié les séjours, de 1958 à 1976, pour en tirer une quinzaine d'articles, et pour finir ce livre unique, conclusif sinon testamentaire, et chargé ainsi d'une importance dépassant largement son propos initial.

Le sujet principal du livre est l'étude et le recensement de ces petites sculptures à figuration humaine, 
monoxyles et en ronde bosse, hautes de 10 à $70 \mathrm{~cm}$, conventionnellement appelées "duka » et qu'il faudrait dénommer, d'après l'auteur, " munga dukna » selon leur désignation vernaculaire à Ndende (sans qu'il précise comment on les désignait par exemple à Vanikoro, où il a pourtant séjourné et fait fabriquer des objets, et où des sculptures de ce type ont été collectées). W. Davenport avait déjà consacré deux articles à ces sculptures, dont il connaissait en 198553 spécimens préservés («A Miniature Figure from Santa Cruz Island », Bulletin des Amis du Musée Barbier-Mueller, $\mathrm{n}^{\circ}$ 28, p. 1), et 53 ou 52 en 1990 («The Figurative Sculpture of Santa Cruz Island » in A. et L. Hanson ed., Art and Identity in Oceania, pp. 99 et 110 , note 1 , avec l'énumération des 52 sculptures !). Le présent ouvrage en reproduit et décrit 55, grâce à la découverte ultérieure de deux spécimens dans les collections du musée de Copenhague, eux aussi collectés à Vanikoro. Ce recensement est au moins incomplet de deux sculptures des collections publiques françaises (Collection Le Mescam, Musée du Havre), provenant également de Vanikoro et d'une poignée d'objets actuellement en mains privées ou sur le marché. La plupart des « duka » reproduits sont présentés comme inédits, parfois par information insuffisante de l'auteur (par exemple $\mathrm{n}^{\circ} 21$ et 22, pp. 150-154, reproduits dans Arts d'Afrique Noire-Arts premiers 102, 1997, pp. 22-23).

Malgré ces menus défauts, le répertoire illustré occupant près de la moitié du livre est le premier du genre et fera date. Il est même remarquable que W. Davenport soit parvenu à en rassembler l'essentiel à une époque où les collections et les inventaires de musées étaient moins faciles d'accès qu'aujourd'hui. On peut regretter que ses notices et ses observations générales restent muettes sur les traces d'outils, l'outillage disponible, les bois mis en œuvre, leur densité ou leur grain, et passent trop rapidement sur les différences de surface ou de patine pourtant si frappantes d'une photographie à l'autre de son catalogue, laissant implicitement ces recherches à ses successeurs, qui devraient les étendre à tous les vestiges subsistants des arts figurés de cet archipel où se sont conjuguées les influences micronésiennes, polynésiennes et mélanésiennes. Il y aurait lieu de soumettre à la même vérification la distinction sommairement avancée (pp. 28-32) entre « generalized » et « developed style » pour des figurations humaines qui avaient des destinations diverses, allant de l'image cultuelle au bouchon historié de gourde à chaux. Mais si cet ouvrage prête à discussion, ce ne saurait être pour la contribution factuelle qu'il apporte à la connaissance iconographique d'un type rare et mal connu de sculpture océanienne.

C'est à partir de ces objets que l'auteur a souhaité livrer une synthèse de ses recherches sur la vie traditionnelle des naturels de Ndende (ou Santa Cruz Island, comme il a toujours préféré écrire à l'usage de son public). Il n'y a rien à redire à une telle ambition, qui fut toujours celle des meilleurs ethnographes, G. Koch notamment. Du rôle rituel de ces sculptures, qui ne se réduisait certainement pas à la représentation d'un unique « dieu » comme l'avait trop rapidement décidé Françoise Girard ( Statuette du Dieu Requin de Santa Cruz», Objets et Mondes XI-3, 1971, pp. 273-280), W. Davenport livre une image plus complexe, mais assez floue. S'agissait-il d'un culte personnel, familial, « clanique » ou encore strictement masculin, ses descriptions « sociologiques » très vagues ne permettent pas de le préciser. Ces figurations avaientelles un rapport avec les usages funéraires et le culte des morts, comme l'avaient fortement suggéré après d'autres Fritz Graebner («Völkerkunde der SantaCruz Inseln », Ethnologica I, 1909, pp. 148-153) et Felix Speiser («Völkerkundliches von Santa Cruz », Ethnologica II, 2, 1916, pp. 205-207), l'ouvrage n'en dit rien, ne mentionne aucun rituel funéraire et se garde de discuter les observations de seconde ou de première main publiées par l'un ou l'autre de ces éminents savants.

Ce n'est pas seulement sur le tard de sa vie que W. Davenport a manifesté son indifférence pour ce que beaucoup nomment «les usages scientifiques», et d'autres "les règles de l'art». Comme j'ai dû me résoudre à le montrer par le menu, notamment à propos des bâtons de danse (« Les bâtons de danse des îles de Santa Cruz et la collection du Musée de Leipzig », Jahrbuch des Museums für Völkerkunde zu Leipzig XLII, 2004, pp. 137-154) et des «monnaies de plumes » de Santa Cruz (étude à paraître), ses articles fréquemment inconséquents ou contradictoires, dans leur détail comme dans leur propos général, négligent ou minimisent régulièrement l'apport des autres témoignages, historiques ou ethnographiques, contemporains ou passés, et omettent les précisions minimales de date et de lieu susceptibles d'authentifier et de distinguer les unes des autres ses observations personnelles. Malgré une bibliographie réduite à l'essentiel, et qu'on pourrait croire connue et exploitée à fond, le présent ouvrage pâtit encore de ces travers, sur lesquels il convient malheureusement de mettre à nouveau en garde les lecteurs.

On lit ainsi (pp. 58-59), à propos des contacts des naturels avec les Blancs, qu'après le passage de la Swallow de Carteret au large de l'archipel de Santa Cruz en 1768, " the next encounter was in 1875 when Commodore James G. Goodenough went ashore at Carlisle Bay near the village of Noka in 1875. He was attacked without provocation and wounded by an arrow. Unfortunately, he contracted tetanus and died on his ship » etc. Plutôt que de lapsus, d'erreur sans conséquence ou d'hommage naïf d'un marin à un autre marin, le sujet porte à parler de cécité aggravée. Goodenough ne fut attaqué qu'en raison d'intrusions répétées de trafiquants en quête d'esclaves, de missionnaires suspects de complicité avec les « blackbirders » et de militaires britanniques en mission de « pacification » ou de représailles : à en croire un historien sacré (C. E. Fox, Lord of the Southern Isles, The Story of the Melanesian Mission, London, 1958, surtout pp. 204 $s q$.) l'évêque Selwyn aurait été calmement accueilli à Ndende en 1852 et 1856, son successeur Patteson de plus en plus fraîchement en 1862 et 1864 , avec la mort de deux membres de son escorte, avant d'être tué lui-même à Nukapu (Reef Islands, à environ $40 \mathrm{~km}$ au nord de Ndende) en 1871. Plusieurs vaisseaux de 
guerre britanniques avaient précédé celui que commandait Goodenough, et notamment en 1865 le Curaçoa où se trouvait Julius L. Brenchley, dont une partie des collectes qu'il avait effectuées au cours de ce voyage, acquise par le British Museum en 1870, a fait l'objet d'un catalogue savant établi par Deborah Waite (Artefacts from the Solomon Islands in the Julius L. Brenchley Collection, British Museum, 1987). C'est à se demander pourquoi ce dernier ouvrage figure dans la bibliographie du livre de Davenport, pour être si nettement démenti dans ses apports les plus incontestables.

De la part d'un ethnologue apparemment épris de la société traditionnelle dont il a tenté de décrire les derniers vestiges, une telle indifférence à l'égard du processus colonial et de ses brutalités laisse pantois. Pour l'historien d'art qu'il entend être aussi, les conséquences ne sont pas négligeables. S'il fallait l'en croire sur l'absence de contacts attestés d'Occidentaux avec les insulaires de Ndende entre le séjour de Mendana en 1595 et la désastreuse tentative de débarquement de Goodenough en 1875, c'est à des collectes espagnoles que devraient être attribués tous les objets de Santa Cruz parvenus entre les mains des Blancs avant 1875, aussi bien ceux qu'a récoltés l'équipage du Curaçoa en 1865 cherchant vainement à punir les deux morts de 1864, que ceux qu'a collectés l'équipage du Rosario, autre vaisseau de guerre britannique affecté à la police coloniale en 1872 (Albert H. Markham, The Cruise of the "Rosario" amongst the New Hebrides and Santa Cruz Islands, exposing the recent atrocities connected with the kidnapping of natives in the South Seas, London, 1873, notamment pp. XVI et 165), ou encore celui qu'il m'est échu de faire surgir des collections du musée d'ethnographie de Leipzig (Me 5483, «Les bâtons de danse » article précité, p. 147 et Taf. XVI), provenant d'une collection rassemblée avant 1839 !

Les autres apports de ce livre souffrent des mêmes faiblesses. Il n'entrait peut-être pas dans les intentions de l'auteur de joindre à ce livre la série de photographies numérisées que reproduit le CD-ROM associé. Ses éditeurs n'ont pas livré mieux qu'un diaporama de retour de vacances, sans date ni lieu, avec des indications génériques moins précises que celles des cartes postales du commerce, et moins utilisables encore pour les spécialistes. Selon ses archives déposées au musée de Berlin, Koch allait jusqu'à noter, outre le lieu, l'heure, la date et parfois l'orientation de la prise de vue, l'appareil utilisé, la focale, le type de pellicule, le diaphragme, la vitesse... Seule l'examen des " archives Davenport » déposées à l'université de Philadelphie permettra de vérifier si l'absence de précisions minimales sur ces photographies est une négligence de l'ethnologue ou de ses éditeurs. En revanche, en n'indiquant que par exception les circonstances dans lesquelles il a collecté les informations qu'il rapporte, c'est décidément le premier qui s'est privé de rendre crédibles ses observations uniques en leur genre concernant la mythologie ou le légendaire des insulaires (pp. 67-94), sur lesquels le reste de la littérature ethnographique est désespérément muet.

Les lecteurs soucieux de tirer parti de ce livre devront ainsi en sonder presque chaque détail. Cer- tains sont vérifiables, telle cette évocation d'un fait divers colonial, « l'affaire Mamuli », où W. Davenport (p. 97) résume sans indication de source le récit qu'en a laissé un ancien District Officer, Hector MacQuarrie (Vouza and the Solomon Islands, New York, 1948, pp. 133-138). Pour ceux qui échappent à la vérification, les plus nombreux et sans doute les plus précieux, il faut s'en remettre à la vraisemblance et à la bonne foi attendue de l'ethnographe. De ce point de vue, inspirée de l'anthropologie behavioriste la plus «moderne», celle qu'il avait apprise à Yale, son œuvre ne se distingue guère des travaux les plus brouillons des folkloristes du XIX ${ }^{\mathrm{e}}$ siècle, et marque une sensible régression par rapport à la « vieille » Wissenschaftlehre historiciste de Koch, dont les résultats, plus limités, restent à toute épreuve. Ses étudiants, ses collègues (et Koch luimême quand nous en avons parlé) ont souligné les qualités personnelles de W. Davenport, sa faconde, sa générosité, sa joie communicative à séjourner dans les îles du Pacifique, et ce livre les laisse quelquefois éclater. Il est regrettable qu'elles n'aient pas porté l'océaniste à léguer aux générations futures, et avant tout aux insulaires de Santa Cruz, un témoignage plus précis sur les vestiges de culture traditionnelle qu'il lui avait été donné de recueillir. Voilà certainement un livre indispensable, mais à utiliser avec la plus grande circonspection.

\section{Gilles BOUNOURE}

William H. Davenport était un des spécialistes les plus éminents de l'art des îles Salomon. Après des études à l' Art Center School de Los Angeles (1939/41), l'auteur découvrit les archipels du Pacifique durant la Deuxième Guerre mondiale alors qu'il était engagé comme lieutenant dans la marine marchande travaillant pour l'us Navy. Après des études d'anthropologie à l'université d'Hawaii, il obtient son Bachelor en 1952, puis un doctorat en ethnologie de l'université de Yale en 1956. Sa carrière se déroula à l'université de Pennsylvanie où il occupa conjointement la chaire de professeur en anthropologie et la charge de conservateur de la section Océanie du musée d'archéologie et d'anthropologie. Il publia de très nombreux articles, tant dans des revues scientifiques que dans des journaux de vulgarisation. Il séjourna vingt-et-un mois, entre 1964 et 1966, aux îles Santa-Cruz situées au sud de l'archipel des Salomon, non loin de l'île de Vanikoro. Il y retourna ensuite épisodiquement pour de courtes périodes, de 1974 à 1976. Cette connaissance du terrain, associée à ses nombreuses relations avec des informateurs locaux et des officiers du Service colonial britannique, lui permit de collecter des informations très précises sur la culture matérielle et l'aida à élaborer une analyse anthropologique de la sculpture dans cette île.

William H. Davenport était sur le point de mettre un terme à l'édition de cet ouvrage, lorsque la mort le surprit en mars 2004. Ce sont donc ses élèves qui se sont chargés de la publication posthume de son manuscrit. Ce dernier reprend en grande partie, tout en le complétant, un précédent article «The Figura- 